Kurt Greiner (2020).

Experimentelle Psychotherapiewissenschaft. Das Methodenprogramm der Wiener Therapieschulenforschung

Berlin: Parados. ISBN: 978-3-9682-4000-8.

176 S., 22,00 EUR, 32,90 CHF

Psychotherapie-Wissenschaft 10 (2) 1052020

www.psychotherapie-wissenschaft.info

CC BY-NC-ND

https://doi.org/10.30820/1664-9583-2020-2-105

Kurt Greiner leitet gemeinsam mit Martin Jandl das Institut für Hermeneutische Therapieschulenforschung \& Therapieschulendialog an der Sigmund Freud PrivatUniversität (SFU) in Wien. Er begründete die Experimental- und Imaginativ-hermeneutische Psychotherapiewissenschaft. Unter dem Titel Experimentelle Psychotherapiewissenschaft stellt er das entsprechende Methodenprogramm an der SFU in Form eines Lehrbuches dar, wie es sich seit 2008 entwickelt hat.

«Gegenstand dieses psychotherapiewissenschaftlichen Programmes sind die spezifischen Funktionsweisen des psychotherapeutischen Denkens und Praxishandelns, deren implizite konzeptuelle Grundlagen, d.h. deren nicht zur Sprache gebrachte Voraussetzungen und ungesehene Bedingungen es in der Experimentellen Psychotherapiewissenschaft mithilfe von speziell entwickelten Reflexionsverfahren und Analysetechniken explizit zu machen bzw. ans Licht zu heben sowie kritisch zu untersuchen gilt. Bei den theorieanalytischen Werkzeugen handelt es sich um neuartige, aussergewöhnliche, teilweise sogar radikal-kreative Instrumentarien des Sinnverstehens (Hermeneutik), die allesamt auf der Idee der 〈Verfremdung〉 im 〈Konstruktiven Realismus〉 (CR) basieren und dem Erkenntnisprinzip des Sinnverstehens durch Sinnverstören folgen» (S. 8).

"Nur durch systematische Veränderung des Gesichtspunktes, also durch Perspektivenverschiebung, kann man es schaffen, die üblichen Vorgehensweisen tatsächlich zum Gegenstand der Betrachtung zu machen. [...] Bei der konstruktiv-realistischen Verfremdung handelt es sich also um den systematischen Versuch, Abstand und Distanz zu den üblichen Denk- und Praxisvollzügen zum eigenen Aktivitätsfeld zu gewinnen» (S. 14).

In vier Abschnitten werden diese Forschungstechniken beschrieben: Standardisierte Therapieschulendialog (TSD) via Experimenteller Trans-Kontextualisation (ExTK); TextPuzzle-Verfahren (P-T-P, ITTP); Psycho-Bild-Methoden (PBP, ITBP, PBS/k, PBS/g); Medien-Spiel-Techniken (PMS, ITMS). Diese vier Techniken werden strukturiert dargestellt und mit Beispielen von Forschungsarbeiten erläutert. So werden im Kapitel «Therapieschulendialog» die anthropologischen Grundlagen der Transpersonalen Psychotherapie mit jenen der Psychoanalytischen Selbstpsychologie nach Heinz Kohut verglichen oder eine transkontextuelle Kon- frontation der Daseinsanalyse (Ludwig Binswanger) mit der Individualpsychologie (Alfred Adler) beschrieben. Im Kapitel «Text-Puzzle-Verfahren» wird das Instanzen- und Trieb-Modell von Sigmund Freud einem Textausschnitt von Carl Rogers gegenübergestellt. Im Kapitel «PsychoBild-Methoden» wird eine Prüfung des transaktionsanalytischen Modells nach Eric Berne dargestellt. Eine weitere bildnerisch verfremdete Prüfung des Instanzenmodells Freuds findet sich im selben Kapitel. Im Kapitel «MedienSpiel-Techniken» finden sich zwei Pilotprojekte zur Untersuchung der Individualtherapie (Alfred Adler) mithilfe der «Psycho-Musik-Analyse», sowie zum Ich-Du-Kontaktbegriff in der Gestalttherapie mit der «Psychotanzanalyse». Im fünften Abschnitt des Buches werden dann Arbeiten zur Experimentellen Psychotherapiewissenschaftspraxis an der SFU mit Stand Februar 2020 aufgelistet.

Das Buch vermittelt einen Eindruck dieser neu geschaffenen Forschungstradition an der SFU und bildet das Spektrum bisheriger Forschungstätigkeiten in diesem speziellen Forschungsfeld ab. Es ist für Aussenstehende etwas schwer zu lesen, weil der Inhalt sehr kompakt dargestellt ist und LeserInnen mit einer Vielzahl neuer und erst einmal fremder Begriffe konfrontiert werden. Dennoch vermag es einen Eindruck zu geben, was hier mit welchen innovativen und erfrischend spielerischen Methoden geforscht wird.

Ich empfehle es allen, die an hermeneutischer Forschung in der Psychotherapiewissenschaft interessiert sind.

Peter Schulthess

\section{Jürgen Kriz (2017). Subjekt und Lebenswelt. Personzentrierte Systemtheorie für Psychotherapie, Beratung und Coaching}

Göttingen: V \& R. ISBN: 978-3-5254-9163-8.

300 S., 30,00 EUR, $36,90 \mathrm{CHF}$

\section{Psychotherapie-Wissenschaft 10 (2) 105-106 2020 \\ www.psychotherapie-wissenschaft.info \\ CC BY-NC-ND \\ https://doi.org/10.30820/1664-9583-2020-2-105b}

Mit diesem sechs Kapitel umfassenden Buch will Jürgen Kriz dazu einladen, sich auf die Komplexität des Geschehens einzulassen, das nun einmal unser Leben als Subjekt in der heutigen Lebenswelt ausmacht. Er ist überzeugt, dass eine grössere Bereitschaft dazu hilft, inhaltliche und theoretische Grabenkämpfe zwischen «Richtungen» zu befrieden, weil die Würdigung für die Perspektive der anderen dann leichter fällt.

Die Personzentrierte Systemtheorie ist aus dem Bedürfnis entstanden, die vielfältigen Prozesse und Einflüsse, die in den unterschiedlichen Ansätzen zu Psychotherapie, Beratung und Coaching jeweils thematisiert werden, in ihrer wechselseitigen Vernetzung zu verstehen. Sie unterscheidet hierzu vier Prozessebenen, die man grob als körperlich, psychisch, interpersonell und kulturell kennzeichnen kann. Mit der Personzentrierten Systemtheorie sollte ein schulenübergreifendes Modell von Psychotherapie 
entwickelt werden, nicht um daraus einen neuen Ansatz zu machen, sondern um das reiche Spektrum der Praxis mit einem solchen Modell besser nutzen zu können.

Im ersten Kapitel führt der Autor in die Grundfragen der Personzentrierten Systemtheorie ein und erläutert die vier Verstehensperspektiven mit dem individualistischpsychodynamisch-humanistischen, dem interpersonellsystemdynamischen, dem organismisch-körperlichen und dem gesellschaftlich-kulturellen Fokus. Er illustriert diese theoretischen Ausführungen mit vier Fallvignetten.

Im zweiten Kapitel folgen einleitend Überlegungen zum Begriff System, um dann die Themen «Subjektivität» und «Objektivität» mit Bezügen zur Philosophie zu vertiefen. Das Kapitel steht unter der Überschrift «Leben als Zeichenprozess - die Perspektive der Biosemiotik». Die Biosemiotik legt eine untrennbare Verwobenheit von psychischen und interpersonellen Phänomenen mit körperlich-evolutionären sowie kulturell-makrosozialen Prozessen nahe. Spannend sind seine Ausführungen zu den Grenzen der Subjektivität, der Bedeutungszuschreibung zu Phänomenen mit Bezügen zur Phänomenologie, der Gestaltpsychologie als Wahrnehmungstheorie und der Experimentellen Psychologie. Auch diese Ausführungen erhalten mit einer Fallvignette einen Praxisbezug.

Im dritten Kapitel werden (essenzielle) Prinzipien der Systemtheorie erläutert. Es geht immer um Prozesse von miteinander dynamisch vernetzten «Teilen». Die Gesamtheit wechselseitig voneinander abhängiger Einflüsse wird als Feld betrachtet. Zu diesen Prinzipien zählt zum Beispiel die Rückkoppelung: Die Veränderung bei einem Teil pflanzt sich im System fort. Oder die Mikro-/Makro-Ebene: Die vernetze und rückgekoppelte Dynamik der «Teile» bildet bottom-up "Ordnungen» aus, die dann top-down die weitere Dynamik der «Teile» bestimmen. Diese Ordnungen sind selbstorganisiert. Oder System versus Umgebung: Selbstorganisation ist zwar etwas dem System Inhärentes, aber immer auch mit Adaptionen an die Systemumgebungen verbunden. Diese Essentials werden in Unterkapiteln wissenschaftstheoretisch mit Bezug zu Forschung und Praxis vertieft. Das Kapitel endet mit einer kurzen zusammenfassenden Darstellung des dynamisch-systemischen in Gegenüberstellung zum klassischen Weltbild.

Im vierten Kapitel werden die Ausführungen zu den eingangs erwähnten vier zentralen Prozessebenen vertieft. Die interpersonelle, die psychische, die gesellschaftlich-kulturelle und die körperliche Prozessebene werden fundiert und anschaulich dargestellt und in ihrem gleichzeitigen Zusammenwirken erläutert. Dies ist mit Exkursen zu Kommunikationstheorie, Gedächtnis, gemeinsamer Kreation von Sinn, und erneut Gestalttheorie sehr spannend zu lesen.

Das füntte Kapitel ist der Welt des Bewusstseins gewidmet. Es enthält eine Besinnung darauf, was mit «Person» gemeint ist. Zentral ist die Aussage, dass «Person» nicht angeboren ist, wohl aber das evolutionär vorstrukturierte Potenzial dazu. Damit sich jemand zur «Person» entfalten kann, braucht es hinreichend guter Entwicklungsbedingungen. Das psychosoziale Beziehungsangebot kann durch drei Kernaspekte einer Grundhaltung beschrieben werden, wie Carl Rogers diese in seinem personzentrierten
Ansatz beschrieben hat: nicht an Bedingungen geknüpfte Wertschätzung des Menschen als Subjekt, kongruentes Angebot von organismischen Erfahrungsmöglichkeiten und deren Symbolisierung, insbesondere durch sprachliche Begleitung, und schliesslich empathisches Einfühlen in die Bedürfnisse des anderen. "Der humanistische Kernsatz: «Nur am Du kann man zum Ich werden` erhält in der Personzentrierten Systemtheorie somit einen umfassenderen Kontext, der u. a. makro- und mikrosoziale, historische oder evolutionäre Perspektiven auf die aktuelle Du-Ich-Begegnung miteinbezieht» (S. 234).

Das sechste Kapitel trägt den Titel «Personzentrierte Systemtheorie im Kontext der Praxis». «Das Anliegen der Personzentrierten Systemtheorie ist es, das grosse Spektrum an vorhandenen Konzepten und Vorgehensweisen in Psychotherapie, Beratung und Coaching besser nutzen zu können» (S. 235). Der Autor erläutert, mit welchen Problemen man eher PsychotherapeutInnen, BeraterInnen oder Coaches bzw. Couchinnen aufsucht. Die professionelle Rolle umfasst als Hauptaufgabe die Begleitung bei «Ordnungs-Ordnungs-Übergängen». Damit sind Transformationen von Ordnungsstrukturen gemeint. Wichtig ist der Gebrauch von Intuition, Imagination und Kreativität.

Das Buch besticht dadurch, dass es nicht Partei nimmt für eine bestimmte psychotherapeutische Richtung, sondern mit der personzentrierten systemischen Sichtweise Bezüge zu vielen «Schulen» herstellt, um zu zeigen, was dort gemacht wird. Kriz beachtet den humanistischen Ethos, dem anderen mit Wertschätzung zu begegnen, wenn er auf andere Therapieansätze eingeht. Wiederholt kritisiert er aber zu Recht den aktuellen Trend zu manualisierten Therapien. Das entspräche nicht dem Bild einer dynamischen, systemischen und prozessorientierten Psychotherapiekonzeption

Subjekt und Lebenswelt behandelt viele grundlegende Themen aus Psychotherapie, Beratung und Coaching fundiert und ist reichlich mit Praxisbeispielen illustriert. In diesen Feldern tätige Menschen finden hier vertiefte Überlegungen und Anregungen zu ihrer Praxistätigkeit und zu deren theoretischen Verankerungen, egal zu welcher Therapierichtung sie sich zählen. Ich kann es gern empfehlen.

Peter Schulthess

\section{Hermann Wegscheider (2020). Dialog und Intersubjektivität in der Gestalttherapie. Von der jüdischen Tradition und Dialogphilosophie zu relationalen Entwicklungen in der Psychoanalyse und Gestalttherapie Gevelsberg: EHP, Verlag Andreas Kohlhage.} ISBN: 978-3-8979-7118-9. 248 S., 26,99 EUR, 37,70 CHF

Psychotherapie-Wissenschaft 10 (2) 106-107 2020

www.psychotherapie-wissenschaft.info

CC BY-NC-ND

https://doi.org/10.30820/1664-9583-2020-2-106

In diesem Buch wird dargelegt, wie manche europäische Therapierichtungen direkt oder indirekt geprägt sind von 